\title{
Bound Magnetopolaron in an Asymmetric Cylindrical Quantum Dot Qubit in an Electric Field
}

\author{
Alain Jerve Fotue ${ }^{1}$, Maurice Tiotsop ${ }^{1}$, Nsangou Issofa ${ }^{1}$, Sadem Christian Kenfack ${ }^{1}$, \\ Amos Virngo Wirngo ${ }^{1}$, Hilaire Fotsin ${ }^{2}$, Lukong Cornelius Fai ${ }^{1}$ \\ ${ }^{1}$ Mesoscopic and Multilayers Structures Laboratory, Department of Physics, Faculty of Science, University of Dschang, Dschang, Cameroon \\ ${ }^{2}$ Laboratory of Electronics and Signal Processing, Department of Physics, Faculty of Science, University of Dschang, Dschang, \\ Cameroon
}

Email address:

fotuea@yahoo.fr (A. J. Fotue)

\section{To cite this article:}

Alain Jerve Fotue, Maurice Tiotsop, Nsangou Issofa, Sadem Christian Kenfack, Amos Virngo Wirngo, Hilaire Fotsin, Lukong Cornelius Fai. Bound Magnetopolaron in an Asymmetric Cylindrical Quantum Dot Qubit in an Electric Field. American Journal of Modern Physics. Vol. 4, No. 3, 2015, pp. 109-117. doi: 10.11648/j.ajmp.20150403.12

\begin{abstract}
We investigate a quantum mechanical system defined as an unsymmetrical bound magnetopolaron immersed in the field of the bulk longitudinal optical (LO)-phonon strong coupling. The ground and the first-excited state of the eigenenergy are derived by using variational method of Pekar type. The effect of the longitudinal and transversal confinement strengths, the effect of magnetic and electric field and the effect of the electron-phonon coupling constant on the polaron characteristics are investigated. These dependencies demonstrate that, they are more flexible tunable methods to restrain quantum decoherence and aggrandize the amplitude of the probability density.
\end{abstract}

Keywords: Magnetopolaron, Quantum Dot, Electric Field

\section{Introduction}

With the exponential advancement of nanotechnology during these last years, the study of quantum computing and quantum information processing has generated widespread interest. The two-level system is usually employed as the elementary unit for storing information. Quantum computation will be based on the laws of quantum mechanics Several schemes have been proposed for realizing quantum computers in recent years [1-9]. For quantum computers to have an edge over classical computers, they will need to carry thousands of qubits. Consequently, these quantum computers with large numbers of qubits will be most feasible as solid-state systems. However, self-assembled quantum dots (QDs) have attracted substantial attention due to their perfect crystal structures. The electric field effects on the energy of confined electrons and holes are studied theoretically in the frame-work of effective-mass envelope function theory,[10-13] which are useful for the application of quantum dots to photoelectric devices. One of the major concerns in QD's is the impurity states, which have attracted extensive attention in recent years [14-18]. More recently, the related problem of an optical polaron bound to a Coulomb impurity in a QD has also been considered in the presence of a magnetic field [19-20]. Imperfections being a rule rather than an exception, such an impurity-bound polaron problem $[21,22]$ is obviously more realistic and is therefore of much practical importance. Wang et al.[23] have recently studied the binding energy of hydrogenic impurities in a GaAs cylindrical QD by using a two-parameter variational wave function. By introducing a trial wave function constructed as a direct product form of an electronic part and a part of coherent phonons, Kandemir et al.[24] have investigated the polaronic effect on the low-lying energy levels of an electron bound to a hydrogenic impurity in a three-dimensional anisotropic harmonic potential subjected to a uniform magnetic field. Chen et al. [25] have investigated the effect of magnetic and electric field on bound polaron in the presence of the hydrogenic impurity; all the work done are not taken into account the two levels system.

In the present paper, the effects of the electron-phonon interaction, the confining potential, the strength of electric and magnetic fields, and the Coulomb potential of hydrogenic impurity on the electron are discussed using the variational method of Pekar type. A single qubit can be envisaged as this kind of two-level quantum system in a QD. 
For such a single-electron QD qubit, Li et al. presented a kind of parameter-phase diagram schemes and defined the parameters region for the use of the $\mathrm{InAs} / \mathrm{GaAs}$ as a twolevel quantum system [26-29]. We have obtained the probability density of the polaron which oscillates with a given period when it is in a superposition of the ground and first excited states. The expressions relating the period of oscillation and the transition frequency of the polaron to the cyclotron frequency, electric field, density parameter and Coulomb potential are derived.

This paper has the following structure: In section 2, we describe the Hamiltonian of the system and use the Pekar variational method to derive the ground and first excited states energy. In section 3, we present results and discussions and finally we end with the conclusion in section 4 .

$$
\begin{aligned}
& H=\frac{1}{2 m}\left(p_{x}-\frac{\beta^{2}}{4} y\right)^{2}+\frac{1}{2 m}\left(p_{y}-\frac{\beta^{2}}{4} x\right)^{2}-e^{*} \rho F+\frac{p_{z}^{2}}{2 m}+\sum_{q} \hbar \omega_{L O} a_{q}^{+} a_{q}+ \\
& +\frac{1}{2} m \omega_{1}^{2} \rho^{2}+\frac{1}{2} m \omega_{2}^{2} z^{2}+\sum_{q}\left[V_{q} a_{q} \exp (i q r)+h . c\right]-\frac{e^{2}}{\varepsilon_{\infty} r}
\end{aligned}
$$

where $\beta^{2}=\left(\frac{2 e}{c}\right) B$ and $m$ is the band mass while $\omega_{1}$ and $\omega_{2}$ are the magnitudes of the transverse and longitudinal confinement strengths of the potentials in the $x y$-plane and the $z$-direction, respectively. $a_{q}^{+}\left(a_{q}\right)$ denotes the creation (annihilation) operator of the bulk $L O$ phonon with wave vector $q, p=\left(p_{x}, p_{y}, p_{z}\right)$ and $r=(\rho, z)$ are the momentum and position vectors of electron and $\rho=(x, y)$ is the position vector of the electron in the $x y-$ plane. $V_{q}$ and $\alpha$ in (2.1) are given as follows:

$$
\begin{aligned}
& V_{q}=i\left(\frac{\hbar \omega_{L O}}{q}\right)\left(\frac{\hbar}{2 m \omega_{L O}}\right)^{1 / 4}\left(\frac{4 \pi \alpha}{V}\right)^{1 / 2} \\
& \alpha=\left(\frac{e^{2}}{2 \hbar \omega_{L O}}\right)\left(\frac{2 m \omega_{L O}}{\hbar}\right)^{1 / 2}\left(\frac{1}{\varepsilon_{\infty}}-\frac{1}{\varepsilon_{0}}\right)^{1 / 2}
\end{aligned}
$$

The Fourier transform of the last expression of the Hamiltonian is written as follow

$$
\frac{1}{r}=\sum_{q} \frac{4 \pi}{V_{q}^{2}} \exp (-i q . r)
$$

To evaluate the energy of our polaron, we use the Pekar variational method. To achieve our goal, we choose the trial function of strong-coupling that can be separated into two parts which individually describe the electron and the phonon. The trial function drawn from [30] is written as:

$$
|\psi\rangle=|\phi\rangle U\left|0_{p h}\right\rangle
$$

\section{Theory and Calculation}

We consider a system in which the electrons are moving in a polar crystal quantum dot with a three-dimensional under the influence of an electric and a magnetic field. The electric field $F$ is along the $\rho$-direction while the magnetic field is along the $z$-direction with vector potential $A=B\left(-\frac{y}{2}, \frac{x}{2}, 0\right)$. The Hamiltonian of the electron-phonon interaction system with a hydrogenic impurity at the center can be written as: harmonic potential and interacting with bulk LO phonons

Where $|\phi\rangle$ depends only on the electron coordinate, $\left|0_{p h}\right\rangle$ represents the phonon's vacuum state with

$a_{q}\left|0_{p h}\right\rangle=0$, and $U\left|0_{p h}\right\rangle$ is the coherent state of the phonon,

$$
U=\exp \left[\sum_{q}\left(a_{\vec{q}} f_{\vec{q}}-a_{\vec{q}} f_{\vec{q}}^{*}\right)\right]
$$

where $f_{\vec{q}}\left(f_{\vec{q}}^{*}\right)$ is the variational function. We may choose the trial ground and the first-excited state wave functions of the electron to be

$$
\begin{array}{r}
\left|\phi_{0}\right\rangle=\left(\frac{2 \lambda_{0}}{\pi}\right)\left(\frac{\mu_{0}}{\pi}\right) \exp \left[-\frac{\lambda_{0} \rho^{2}}{2}\right] \exp \left[-\frac{\mu_{0} z^{2}}{2}\right] \\
\left|\phi_{1}\right\rangle=2\left(\frac{\lambda_{1}}{\pi}\right)^{1 / 2} \frac{\mu_{1}^{3 / 4}}{\pi^{1 / 4}} z \exp \left[-\frac{\lambda_{1} \rho^{2}}{2}\right] \exp \left[-\frac{\mu_{1} z^{2}}{2}\right]
\end{array}
$$

where $\lambda_{0}, \mu_{0}, \lambda_{1}$ and $\mu_{1}$ are the variational parameters. Equations (2.5) and (2.6) satisfy the following normalized relations:

$$
\left\langle\phi_{0} \mid \phi_{0}\right\rangle=\left\langle\phi_{1} \mid \phi_{1}\right\rangle=1 ;\left\langle\phi_{0} \mid \phi_{1}\right\rangle=0
$$

Using the Pekar variational method, we have

$$
H^{\prime}=U^{-1} H U
$$

By minimizing the expectation value of the Hamiltonian, that is

$$
E_{0}=\left\langle\phi_{0}\left|H^{\prime}\right| \phi_{0}\right\rangle \text { and } E_{0}=\left\langle\phi_{1}\left|H^{\prime}\right| \phi_{1}\right\rangle \text {, we then obtain }
$$


the magnetopolaron ground and first excited state energy in the following forms:

$$
\begin{aligned}
& E_{0}=\lambda_{0}+\frac{\mu_{0}}{2}+\frac{1}{\lambda_{0} l_{1}^{4}}+\frac{1}{2 \mu_{0} l_{2}^{4}}++\frac{\omega_{c}^{2}}{16 \lambda_{0}}-\alpha \sqrt{\frac{2 \lambda_{0}}{\pi\left(1-\frac{\lambda_{0}}{\mu_{0}}\right)}} \arcsin \left(1-\frac{\lambda_{0}}{\mu_{0}}\right)^{1 / 2}- \\
& -\frac{\sqrt{\pi} e^{*} F}{2 \sqrt{\mu_{0}}}-2 \gamma \sqrt{\frac{\lambda_{0}}{\pi\left(1-\frac{\lambda_{0}}{\mu_{0}}\right)}} \arcsin \left(1-\frac{\lambda_{0}}{\mu_{0}}\right)^{1 / 2}
\end{aligned}
$$

And

$$
\begin{aligned}
& E_{1}=\lambda_{1}+\frac{3 \mu_{1}}{2}+\frac{1}{\lambda_{1} l_{1}^{4}}+\frac{3}{2 \mu_{1} l_{2}^{4}}++\frac{\omega_{c}^{2}}{16 \lambda_{1}}-\alpha \sqrt{\frac{2 \lambda_{1}}{\pi\left(1-\frac{\lambda_{1}}{\mu_{1}}\right)}} \arcsin \left(1-\frac{\lambda_{1}}{\mu_{1}}\right)^{1 / 2}- \\
& -\frac{\sqrt{\pi} e^{*} F}{2 \sqrt{\mu_{1}}}-2 \gamma \sqrt{\frac{\lambda_{1}}{\pi\left(1-\frac{\lambda_{1}}{\mu_{1}}\right)}} \arcsin \left(1-\frac{\lambda_{1}}{\mu_{1}}\right)^{1 / 2}
\end{aligned}
$$

Where $l_{1}=\sqrt{\frac{\hbar}{m \omega_{1}}}, \quad l_{2}=\sqrt{\frac{\hbar}{m \omega_{2}}}$ are the transverse and longitudinal effective confinement lengths of the QD, respectively. $\gamma=\frac{e^{2}}{\varepsilon_{\infty}}$ is the Coulomb potential

The superposition state of electron can be expressed as:

Where

$$
|0\rangle=\phi_{0}(\rho, z)=\left(\frac{2 \lambda_{0}}{\pi}\right)\left(\frac{\mu_{0}}{\pi}\right) \exp \left(-\frac{\lambda_{0} \rho^{2}}{2}\right) \exp \left(-\frac{\mu_{0} z^{2}}{2}\right)
$$

And

$$
|1\rangle=\phi_{1}(\rho, z)=2\left(\frac{\lambda_{1}}{\pi}\right)^{1 / 2} \frac{\mu_{1}^{3 / 4}}{\pi^{1 / 4}} z \exp \left[-\frac{\lambda_{1} \rho^{2}}{2}\right] \exp \left[-\frac{\mu_{1} z^{2}}{2}\right]
$$

The time evolution of the state of the electron can then be written as

$$
\psi_{01}(t, \rho, z)=\frac{1}{\sqrt{2}} \phi_{0}(\rho, z) \exp \left(-\frac{i E_{0} t}{\hbar}\right)+\frac{1}{\sqrt{2}} \phi_{1}(\rho, z) \exp \left(-\frac{i E_{1} t}{\hbar}\right)
$$

The probability density is in the following form:

$$
\begin{aligned}
Q(t, \rho, z) & =\left|\psi_{01}(t, \rho, z)\right|^{2} \\
& =\frac{1}{\sqrt{2}}\left[\begin{array}{l}
\left|\phi_{0}(\rho, z)\right|^{2}+\left|\phi_{1}(\rho, z)\right|^{2}+\phi_{0}^{*}(\rho, z) \phi_{1}(\rho, z) \exp \left(i \omega_{01} t\right)+ \\
+\phi_{0}(\rho, z) \phi_{1}^{*}(\rho, z) \exp \left(-i \omega_{01} t\right)
\end{array}\right]
\end{aligned}
$$

Where is the transition frequency from ground state to the first level.

$$
\omega_{01}=\frac{\left(E_{1}-E_{0}\right)}{\hbar}
$$

\section{Results and Discussions}

In this part, we show the numerical results of the ground 
and first excited state energies, the transition frequency, the probability density and the period of oscillation versus the electron-phonon coupling strength, the cyclotron frequency and the electric strength parameter.

In figures 1-2, we have plotted the ground and first excited state energy and transition frequency as a function of electron-phonon coupling strength $\alpha$ for fixed $l_{1}=0.25$, $F=2.0 ; \omega_{c}=5.0$ and $\beta=0.5 \quad$ (figure 1) and for $l_{1}=0.60$ (figure 2 ). These figures clearly illustrate that $E_{0}, E_{1}$ and $\omega$ are increasing functions of the electron-phonon coupling strength $\alpha$. Thus the state energies and the transition frequency of an Anisotropic QDs can be controlled by those parameters. In qubits, the electron-phonon interaction strength will increase when the system is confined, resulting in a larger transition frequency and the destroying of the superposition state (decoherence). The present result of controlling the superposition state by tuning the state energies and the transition frequency may have practical usage in quantum information processes. This is the third way by which the decoherence process can be eliminated, i.e., crystal materials with smaller coupling strength for the design of an anisotropic QDs.

In figures 3-6, we have plotted the ground and first excited states polaron energy as a function of electric strength $F$ (figure 3), coulomb potential $\beta$ (figure 4) and cyclotron frequency $\omega_{c}$ (figure 5). In figure 6 , we have plotted the transition frequency as a function of electric field strength $F$ (figure 6(a)), cyclotron frequency $\omega_{c}$ (figure $6(\mathrm{~b})$ ) and coulomb potential $\beta$ (figure 6(c)). From figure 3, we see that the ground and first excited state energy are the decreasing function the electric field strength. When the electric field becomes stronger, the electron moves away from the center and gets closer to the surface along the axis, resulting in the contribution of the bulk LO phonon to the binding energy being decreased, which are very important experimentally to control and modulate the intensity of optoelectronic devices. From figure 5, it's obvious that the ground and first excited states energies are the increase function of cyclotron frequency. Since the presence of a magnetic field is equivalent to introducing another confinement on electrons which leads to greater overlap of the electron wave function, the electron-phonon interactions will be enhanced, and the ground and first excited states binding energies appears more obvious.

Figs. 3 and 5 also illustrate that the ground and first excited states binding energy increases with increasing confinement strengths in the lateral and the longitudinal directions. This result is consistent with that of Lepine [31] and Chen [25]. From figure 4, it is obvious that the ground state binding energy is an increasing function of the Coulomb potential [25].

The transition frequency is the increasing function of the electric field strength, cyclotron frequency, Coulomb potential and longitudinal effective confinement lengths (figure 6). Thus, the electron energy, the electron-phonon interactions, the ground and first excited state energies and the transition frequency increase with the increasing magnetic field and impurity parameter. These results are in agreement with the results of Kandemir and Cetin [30] and those of Wei Xiao and Jing-Lin Xiao[1].

Figures 7-9 show the spatiotemporal evolution of the polaron probability density $\left|\psi_{01}(t, \rho, z)\right|^{2}$ when the polaron exists in the superposition state of $\left|\psi_{01}\right\rangle=\frac{1}{\sqrt{2}}(|0\rangle+|1\rangle)$ for those parameters

$$
\begin{gathered}
l_{1}=0.75 \quad ; l_{2}=0.80 ; \quad \omega_{c}=0.5 ; \\
\alpha=6.5 \quad F=1.5 \quad \text { and } \beta=0.5 \\
l_{1}=0.75 \quad ; l_{2}=0.80 ; \quad \omega_{c}=3.0 ; \\
\alpha=6.5 \quad F=1.5 \quad \text { and } \beta=0.5 \\
l_{1}=0.75 \quad ; l_{2}=0.80 ; \quad \omega_{c}=1.5 ; \\
\alpha=6.5 \quad F=1.5 \quad \text { and } \beta=0.5 \\
l_{1}=0.75 \quad ; l_{2}=0.80 ; \quad \omega_{c}=1.5 ; \\
\alpha=6.5 \quad F=1.5 \quad \text { and } \beta=2.0 \\
l_{1}=0.75 \quad ; l_{2}=0.80 ; \quad \omega_{c}=2.5 ; \\
\alpha=6.5 \quad F=0.5 \quad \text { and } \beta=0.5 \\
l_{1}=0.75 \quad ; l_{2}=0.80 ; \quad \omega_{c}=2.5 ; \\
\alpha=6.5 \quad F=1.5 \quad \text { and } \beta=0.5
\end{gathered}
$$

The probability density increases with a decrease of the field strength, Coulomb potential. Near $\rho=0$ the density has its maximal value. The probability density of the electron oscillates with a period of oscillation $\tau_{0}=\frac{\hbar}{\left(E_{1}-E_{0}\right)}$ this means that the information is transferred from one state to another.

Figure 10 (a) is a plot of the period of oscillation of the polaron in a quantum dot as a function of electric field strength for fixed $l_{1}=0.5$ and different $\alpha$, figure 10 (b) is a plot of the period of oscillation of the polaron in a quantum dot as a function of the cyclotron frequency for fixed $l_{1}=0.5$ and different $\alpha$ and figure 10 (c) is a plot of the period of oscillation of the polaron in a quantum dot as a function of Coulomb potential for fixed $l_{1}=0.5$ and different $\alpha$. It is obvious from here that the period of oscillation is a decreasing function of the electric field, magnetic field and Coulomb potential. As a result of the electric field, magnetic field and Coulomb impurity, the ground and the first-excited state energies increase and the influence is greater on the first-excited state energy. The period of oscillation $\tau_{0}$ decreases, that is to say the life time of a qubit reduces, so the process of decoherence is quickened [32-34]. It can be very harmful to store information with the QD as its elementary unit. 


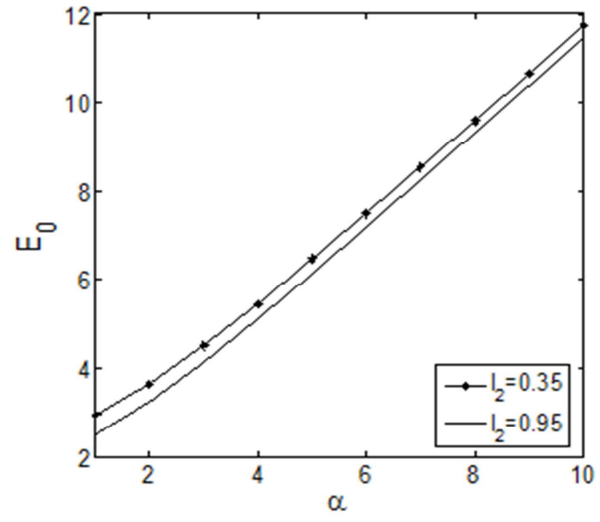

(a)

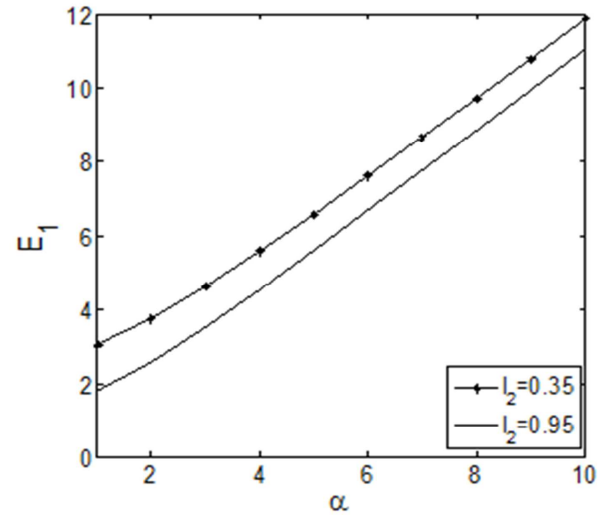

(b)

Figure 1. (a) Ground state energy $E_{0}$ in function of the electron-phonon coupling strength $\alpha$ for fixed $l_{1}=0.25, F=2.0 ; \omega_{c}=5.0$ and $\beta=0.5$. (b) First excited state energy $E_{1}$ in function of the electron-phonon coupling strength $\alpha$ for fixed $l_{1}=0.25, F=2.0 ; \omega_{c}=5.0 \quad$ and $\beta=0.5$

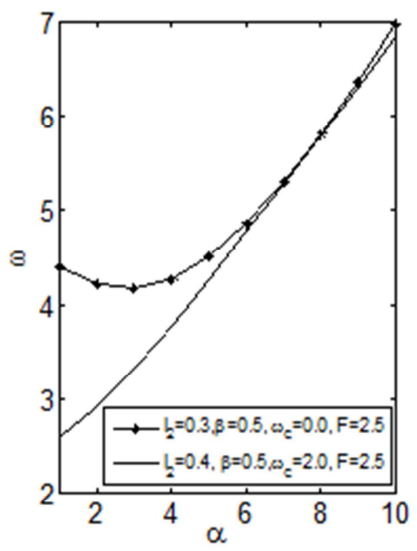

(a)

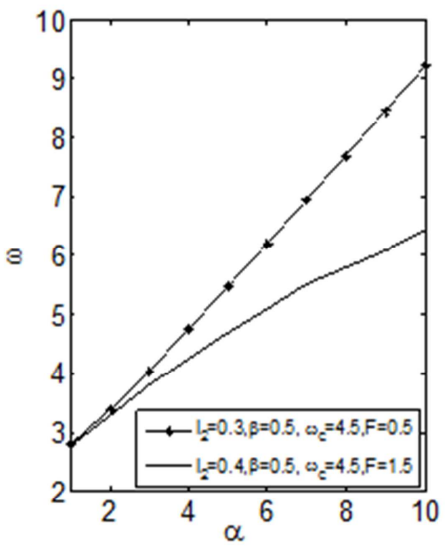

(b)

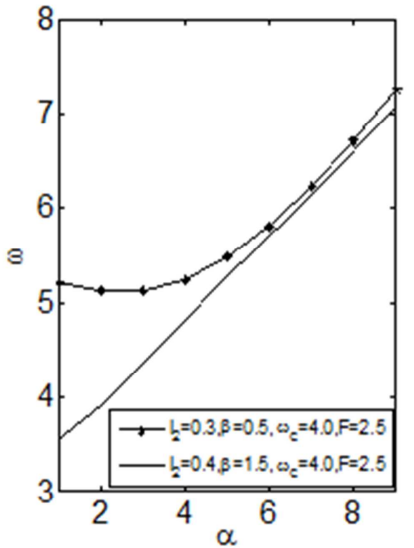

(c)

Figure 2. (a)Transition frequency $\omega$ in function of electron-phonon coupling constant $\alpha$ for fixed $l_{1}=0.6 ; F=2.5$ and $\beta=0.5$. (b)Transition frequency $\omega$ in function of electron-phonon coupling constant $\alpha$ for fixed $l_{1}=0.6 ; \omega_{c}=4.5$ and $\beta=0.5$, (c) Transition frequency $\omega$ in function of electronphonon coupling constant $\alpha$ for fixed $l_{1}=0.6 ; F=2.4$ and $\omega_{c}=4.0$

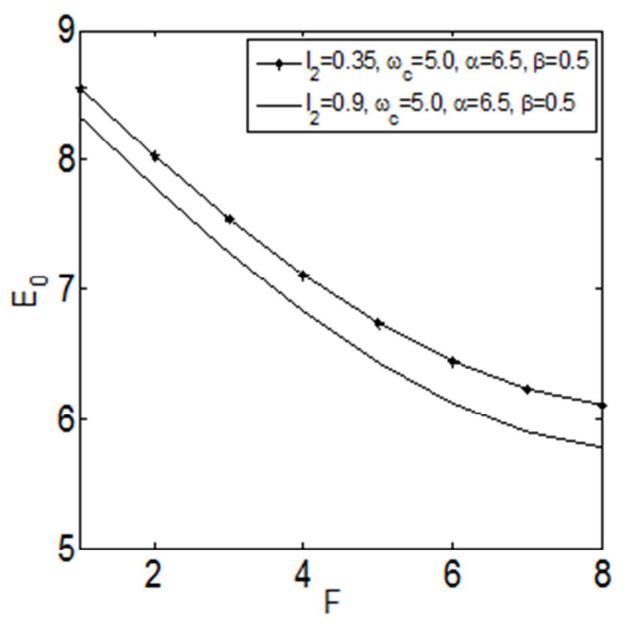

(a)

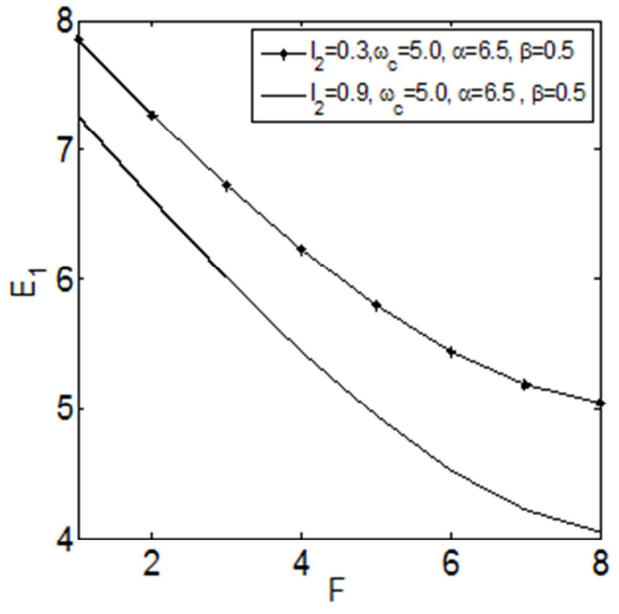

(b)

Figure 3. Ground state and first excited state energy as a function of the electric field strength $F$ for fixed $l_{1}=0.25$. 


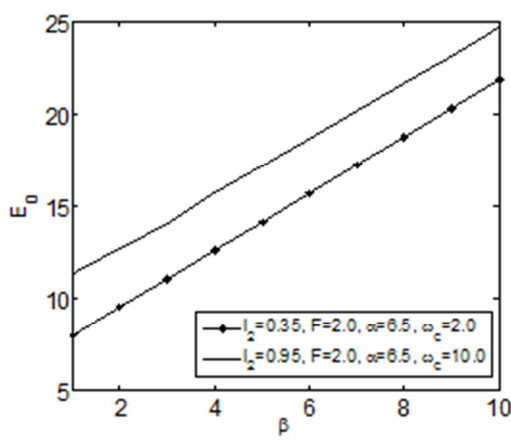

(a)

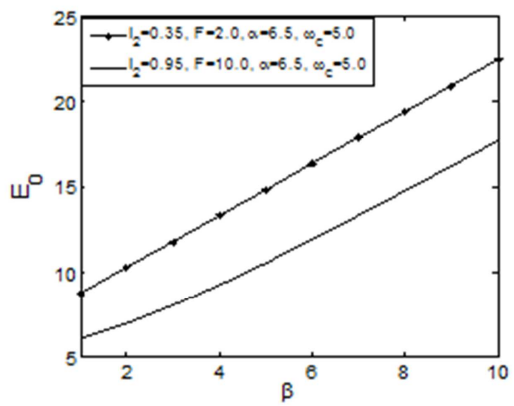

(c)

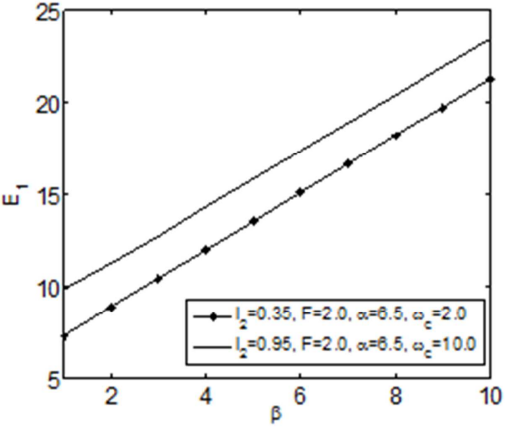

(b)

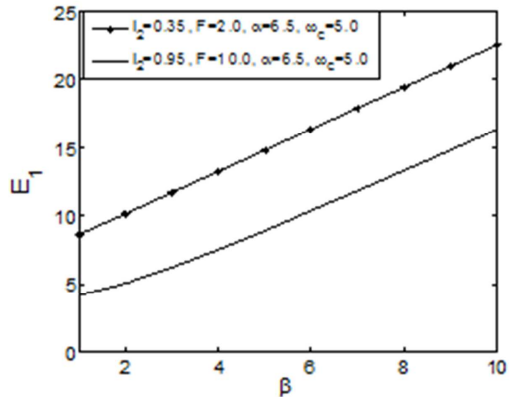

(d)

Figure 4. Ground state and first excited state energy as a function of the Coulomb potential $\beta$ for fixed $l_{1}=0.25$.

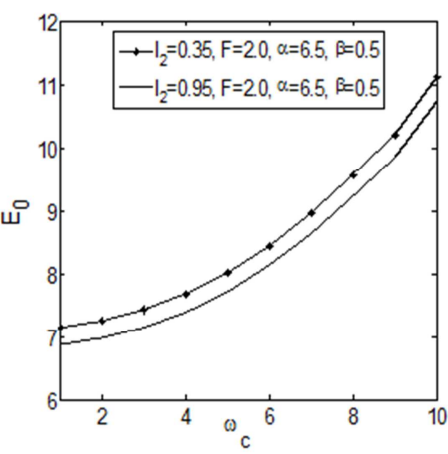

(a)

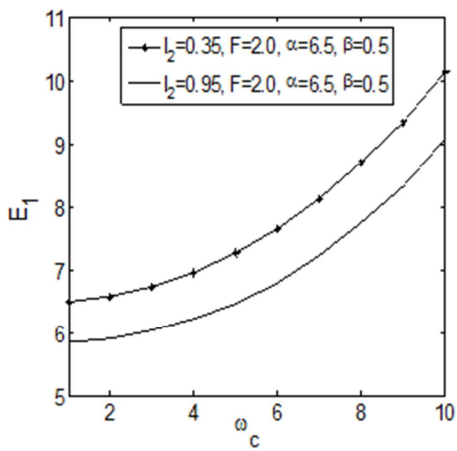

(b)

Figure 5. Ground state and first excited state energy as a function of the cyclotron frequency $\omega_{c}$ for fixed $l_{1}=0.25$.
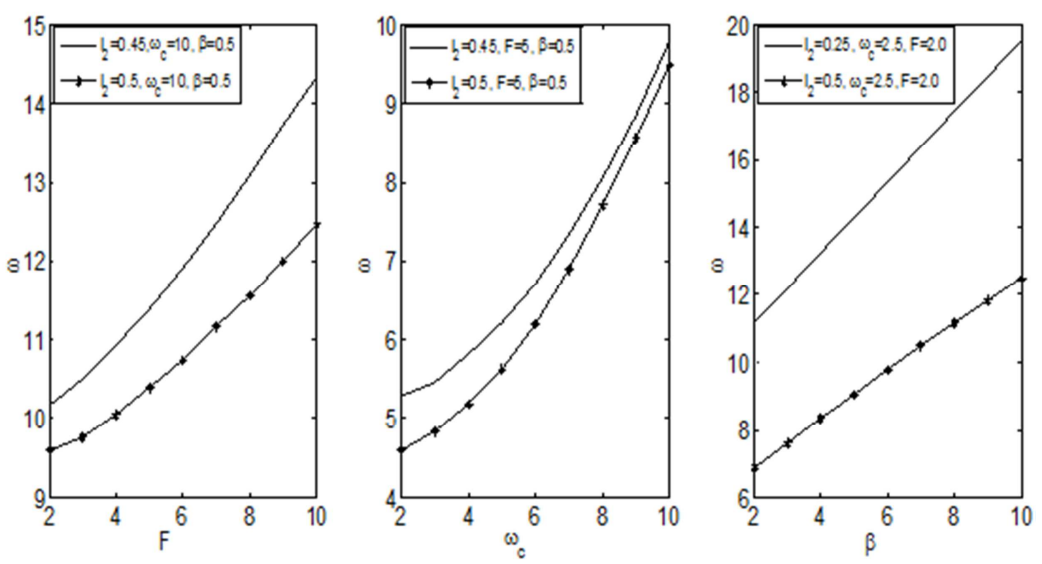

Figure 6. (a) Transition frequency $\omega$ in function of electric field strength $F$ for fixed $l_{1}=0.45 ; \omega_{c}=10 ; \alpha=6.5$ and $\beta=0.5$ (b) Transition frequency

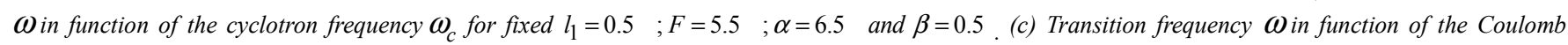
potential $\beta$ for fixed $l_{1}=0.5 ; F=2.5 ; \alpha=6.5$ and $\omega_{c}=1.5$ 

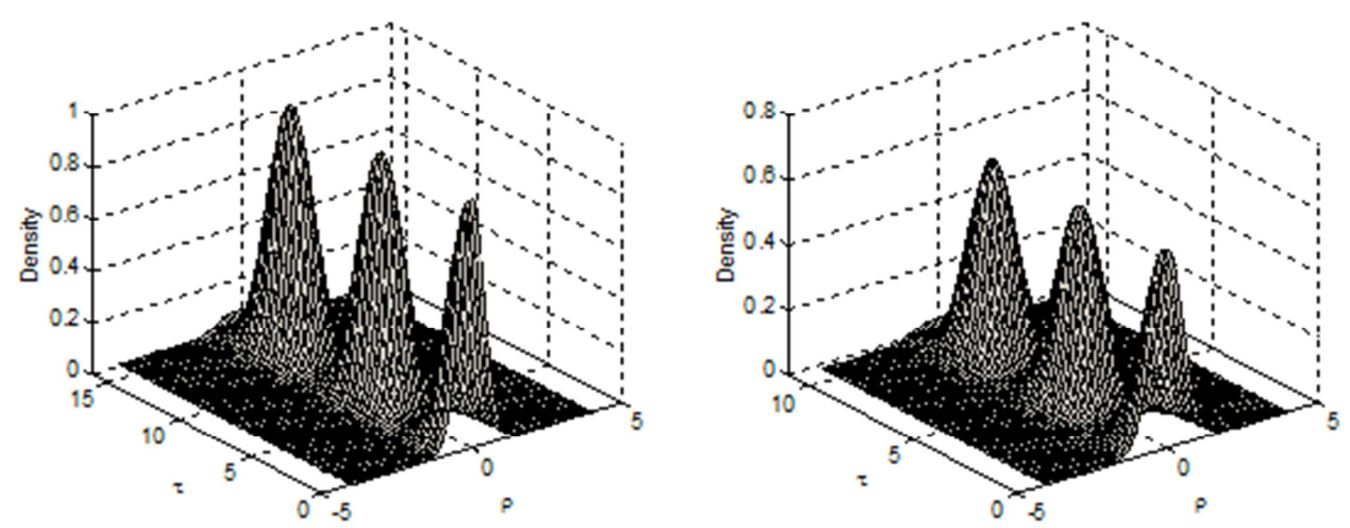

Figure 7. (a) Spatiotemporal evolution of the electron probability density in superposition state of $|0\rangle$ and $|1\rangle$ for $l_{1}=0.75 ; l_{2}=0.80 ; \quad \omega_{c}=0.5 ; \alpha=6.5 \quad F=1.5 \quad$ and $\beta=0.5 ;$ (b) Spatiotemporal evolution of the electron probability density in superposition state of $|0\rangle$ and $|1\rangle$ for $l_{1}=0.75 \quad ; l_{2}=0.80 ; \quad \omega_{c}=3.0 \quad ; \alpha=6.5 \quad F=1.5 \quad$ and $\beta=0.5$
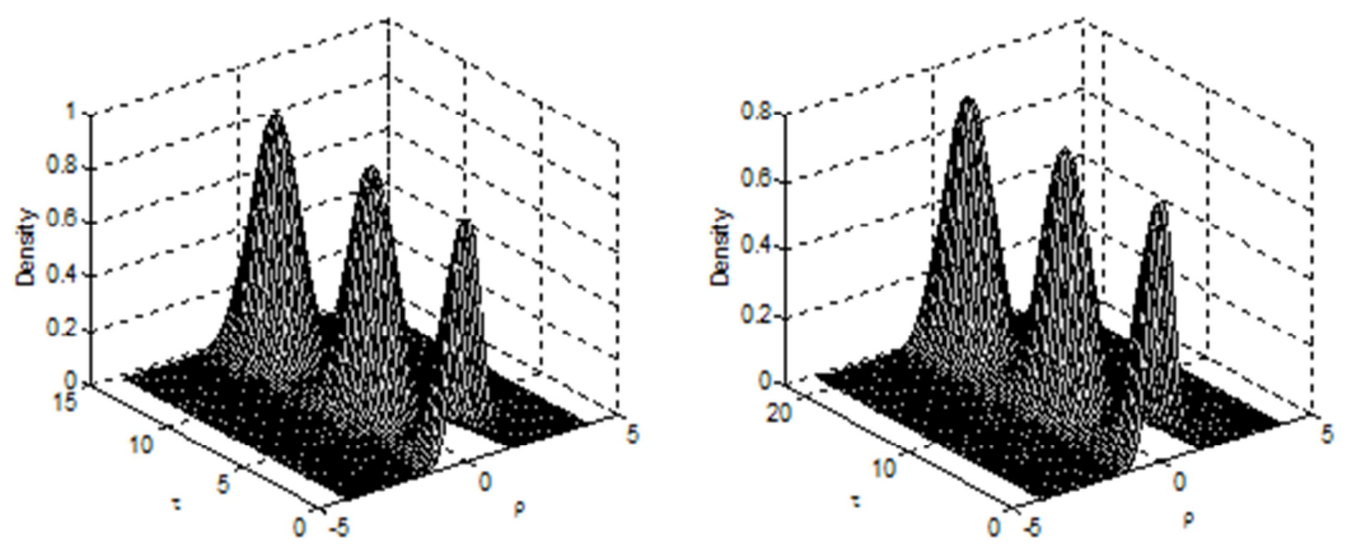

Figure 8. (a) Spatiotemporal evolution of the electron probability density in superposition state of $|0\rangle$ and $|1\rangle$ for $l_{1}=0.75 ; l_{2}=0.80 ; \quad \omega_{c}=1.5 ; \alpha=6.5 \quad F=1.5 \quad$ and $\beta=0.5 \quad$ (b) Spatiotemporal evolution of the electron probability density in superposition state of $|0\rangle$ and $|1\rangle$ for $l_{1}=0.75 ; l_{2}=0.80 ; \quad \omega_{c}=1.5 \quad ; \alpha=6.5 \quad F=1.5 \quad$ and $\beta=2.0$
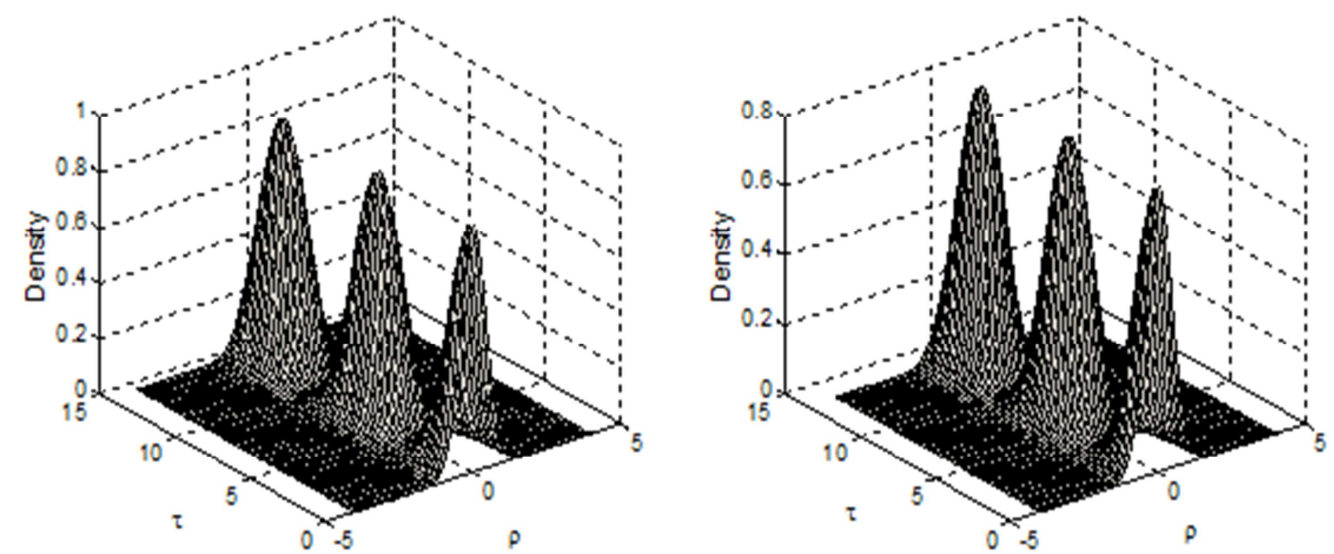

Figure 9. (a) Spatiotemporal evolution of the electron probability density in superposition state of $|0\rangle$ and $|1\rangle$ for $l_{1}=0.75 ; l_{2}=0.80 ; \quad \omega_{c}=2.5 ; \alpha=6.5 \quad F=0.5 \quad$ and $\beta=0.5 \quad$ (b) Spatiotemporal evolution of the electron probability density in superposition state of $|0\rangle$ and $|1\rangle$ for $l_{1}=0.75 \quad ; l_{2}=0.80 ; \quad \omega_{c}=2.5 ; \alpha=6.5 \quad F=1.5 \quad$ and $\beta=0.5$. 


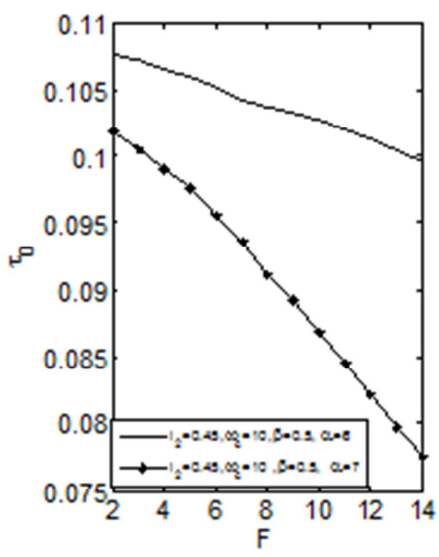

(a)

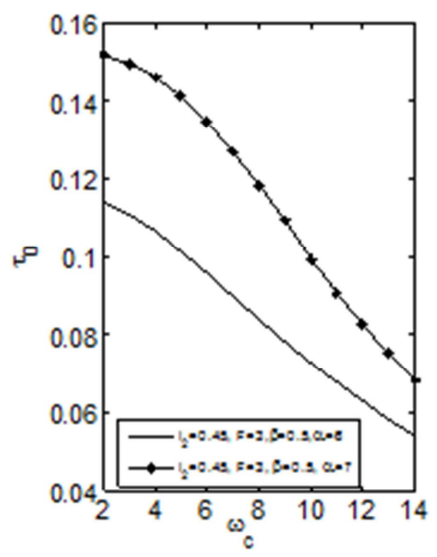

(b)

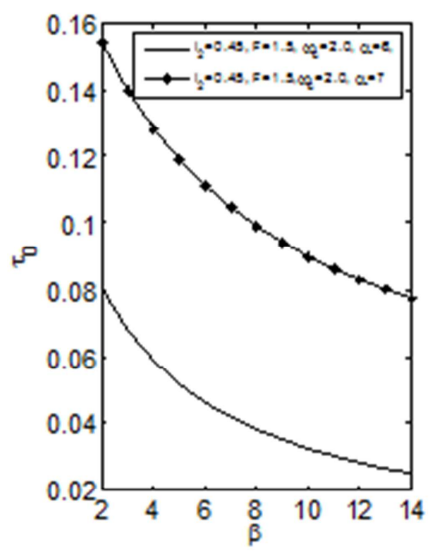

(c)

Figure 10. (a) The period of oscillation of polaron in a quantum dot as a function of the electric strength $F$ for fixed $l_{1}=0.5$ and different $\alpha$, (b) The period of oscillation of polaron in a quantum dot as a function of the cyclotron frequency $\omega_{c}$ for fixed $l_{1}=0.5$ and different $\alpha$; (c) The period of oscillation of polaron in a quantum dot as a function of the Coulomb potential $\beta$ for fixed $l_{1}=0.5$ and different $\alpha$.

\section{Conclusion}

In this paper, we have derived the ground and first excited state energies of the bound magnetopolaron under an electric, magnetic field and Coulomb potential in an asymmetric quantum dot and their relevant eigen-functions using the Pekar variational method. The single qubit can be envisaged as this kind of two-level quantum system in QDs. The probability density of electron oscillates with a period when the polaron is in the superposition state of the ground and the first-excited state. The probability density of the polaron increases with a decrease of the electric field, magnetic field strength and Coulomb potential in the superposition state while the period of oscillation decreases with increasing electric field magnetic field strength and Coulomb potential. Our results should be meaningful for designing the solid-state implementation of quantum computing both theoretically and experimentally and also for the control of decoherence in quantum system.

\section{References}

[1] W. P. Li, J. W. Yin, Y. F. Yu, Z.W. Wang and J.L. Xiao, J. Low Temp. Phys. 160 112(2010)

[2] Y. F. Yu, W.P. Li, J. W. Yin and J.L. Xiao, J. Low Temp. Phys. $503322(2011)$

[3] Y. J. Wen, J. L. Xiao, Y. F. Yu, Z. W. Wang, Chinese Phys. B 18446 (2009)

[4] S. Jia-kui, L. Hong-Juan, J. L. Xiao, Physica B 404 1961(2009)

[5] M. A. Nielsen, I. L. Chang, Quantum Computation and Quantum Information (Cambridge University Press, Cambridge, 2000)

[6] I. D'Amico, Microelectron. J. 37 1440(2006)

[7] C. H. Bennett, D. P. DiVincenzo, Nature 404247 (2000)
[8] P. Hawrylak, M. Korkusinski, Solid State Commun 136508 (2005)

[9] S. D. Sarma, R.D.Sousa, X. D. Hu, B. Koiller, Solid State Commun. 133 737(2005)

[10] M. A. Kastner, Rev. Mod. Phys. 64849 (1992)

[11] M. W. Dellow, P. H. Beton, C. J.G. M. Langerak, T. J. Foster, P. C. Main, L. Eaves, M. Henini, S. P. Beaumont and C. D. W. Wilkinson, Phys. Rev. Lett.68, 1754 (1992)

[12] S. S. Li and J. B. Xia, Phys. Rev. B 583561 (1998)

[13] S. S. Li and J. B. Xia, Appl. Phys. Lett. 87043102 (2005)

[14] C. Sikorski and U. Merkt, Phys Rev. Lett. 622164 (1989)

[15] S.S. Li and J. B. Xia, Phys. Lett. A 366120 (2007)

[16] A. Lorke, J. P. Kotthaus and K. Ploog, Phys. Rev. Lett. 64 2559(1990)

[17] X.H Qi, X. J. Kong and J.J. Liu, Phys. Rev. B , Condens. Matter 58(16) 10578 (1998)

[18] S. S. Li and J. B. Xia, J. Appl. Phys. 100083714 (2006)

[19] K. D. Zhu and T. Kobayashi, Solid State Commun. 92 353(1994)

[20] T. C. Au-Yeung, S. L. Kho, S. W. Gu, L. H. Hong and E. M. C. Wong, J. Phys.: Condens. Matter 66761 (1994)

[21] S. Sil and A. Chatterjee, J. Phys. Condens. Matter 3 9401(2001)

[22] M. Bhattacharya, A. Chatterjee and T. K. Mitra, Phys. Rev. B 398351 (1989)

[23] R. Q. Wang, H. J. Xie and Y. B. Yu, Phys. Stat. Sol. B 242890 (2005)

[24] B. S. Kandemir and A. Cetin, Phys. Rev. B 65 054303(2002).

[25] S. H. Chen and J.L. Xiao, Int. J. of Mod. Phys. B 22 162611(2008).

[26] J. L. Xiao, J. Low Temp Phys 172 122(2013). 
[27] S.S. Li, J. B. Xia, F. H. Yang, Z. C. Niu, S. L. Feng, H. Z. Zheng, J. Appl. Phys. 90 6151(2001)

[28] S. S. Li, L. Gui- Lu, F. S. Bai, S. L. Feng, H. Z. Zheng, Proc. Natl. Acad. Sci. USA 98 11847(2001).

[29] Z. W. Wang, J.L. Xiao, Acta Phys. Sin. 56678 (2007).

[30] B. S. Kandemir and A. Cetin, J. phys. condens. Matter 17 667(2005).

[31] Y. Lepine and G. Bruneau, J. Phys. Condens. Matter 10 1495(1998).
[32] D. Tolkunov, V. Privman, Phys. Rev. A 69062309 (2004).

[33] A. Grodecka, P. Machnikowski, Phys. Rev. B 73125306 (2006).

[34] M. Lovric , H. G. Krojanski, D. Suter, Phys. Rev. A 75042305 (2007). 